\title{
Natural history of apical hypertrophic cardiomyopathy and novel surgical treatment
}

\author{
Kashish Goel, MBBS, ${ }^{a}$ Hartzell V. Schaff, MD, ${ }^{b}$ and Rick A. Nishimura, MD ${ }^{a}$
}

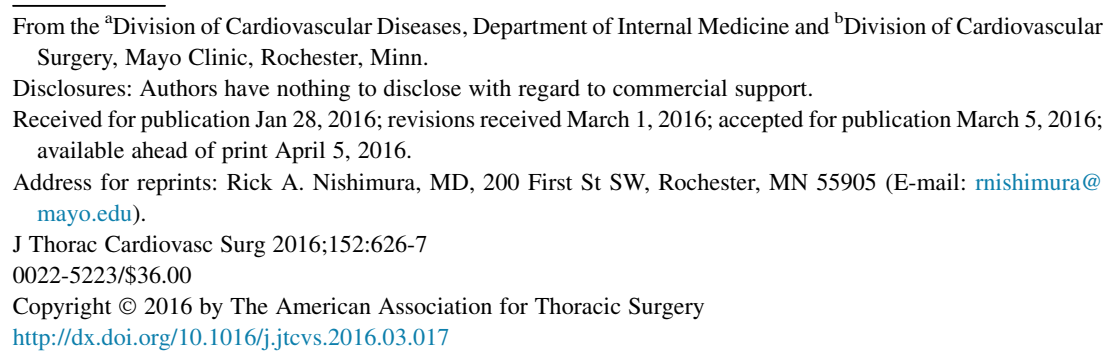

A 47-year-old woman with hypertrophic cardiomyopathy was referred for further evaluation. Twenty-five years earlier, a left ventriculogram had shown a "spade-like" configuration of the left ventricular (LV) cavity in systole (Figure 1, A) and diastole (Figure 1, B), confirming the diagnosis of apical hypertrophic cardiomyopathy. The patient had done well until 4 years ago, when she noticed palpitations and exertional dyspnea. One year ago, she experienced 2 episodes of transient ischemic attack. She was subsequently found to have both atrial fibrillation and nonsustained ventricular tachycardia. She underwent radiofrequency ablation, restoring normal sinus rhythm. However, she continued to experience severe dyspnea and her cardiopulmonary exercise test revealed severe cardiac output limitation. Normal coronary arteries were present on coronary angiography.

Cardiac magnetic resonance imaging (MRI) revealed an apical aneurysm and complete obliteration of the midventricular cavity (Figure 2). For persistent symptoms and thromboembolic events, she underwent apical ventriculotomy, removal of an apical thrombus, resection of the apical pouch,

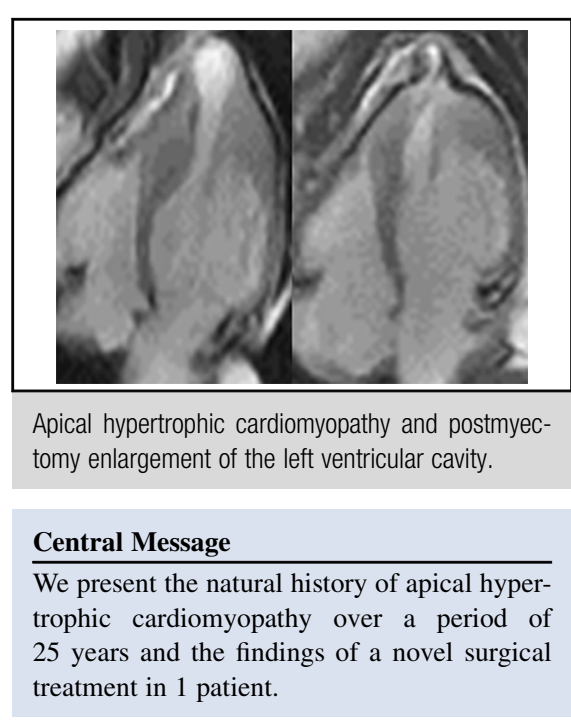

See Editorial Commentary page 628.

and extensive midventricular myectomy. Postoperative cardiac MRI showed complete resolution of midventricular obliteration and resection of the apical aneurysm (Figure 3, A) and enlargement of the LV cavity in diastole (Figure 3, $B$ ). The patient had an improvement in symptoms with no further ventricular ectopy or embolic events.

These images illustrate the natural history of apical hypertrophic cardiomyopathy over a 25 -year period. Apical aneurysms are present in $2 \%$ to $3 \%$ of patients with
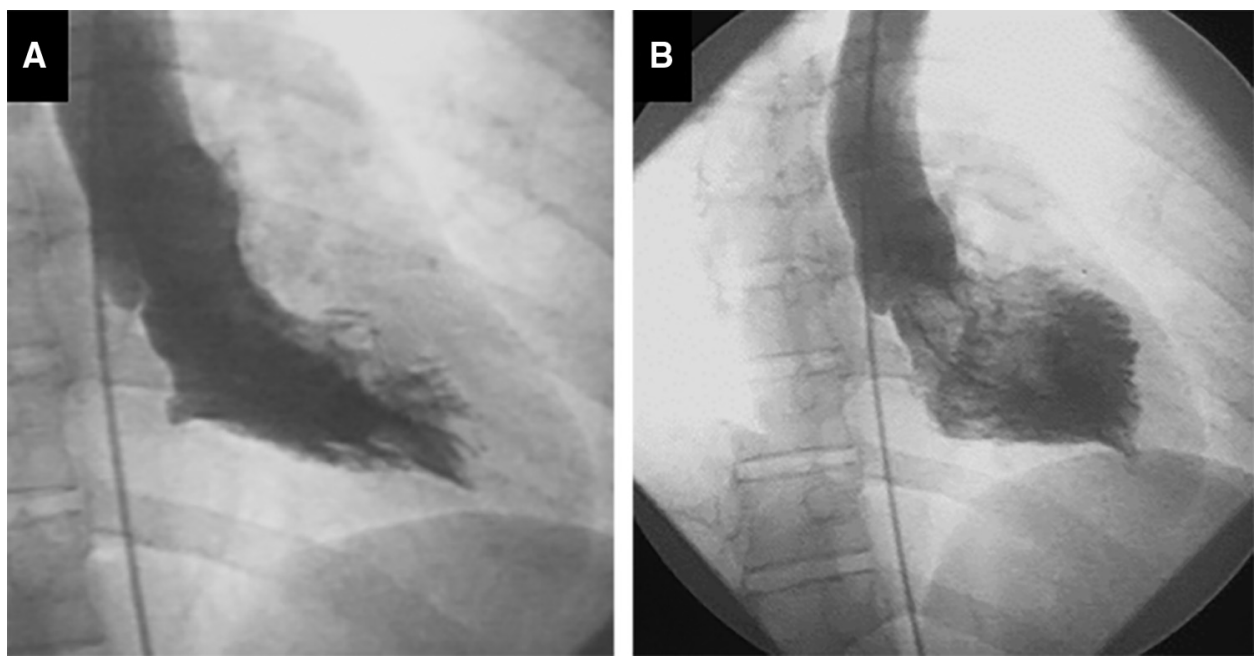

FIGURE 1. Left ventriculogram in (A) systole and (B) diastole, showing the early stages of apical aneurysm formation in hypertrophic cardiomyopathy. 

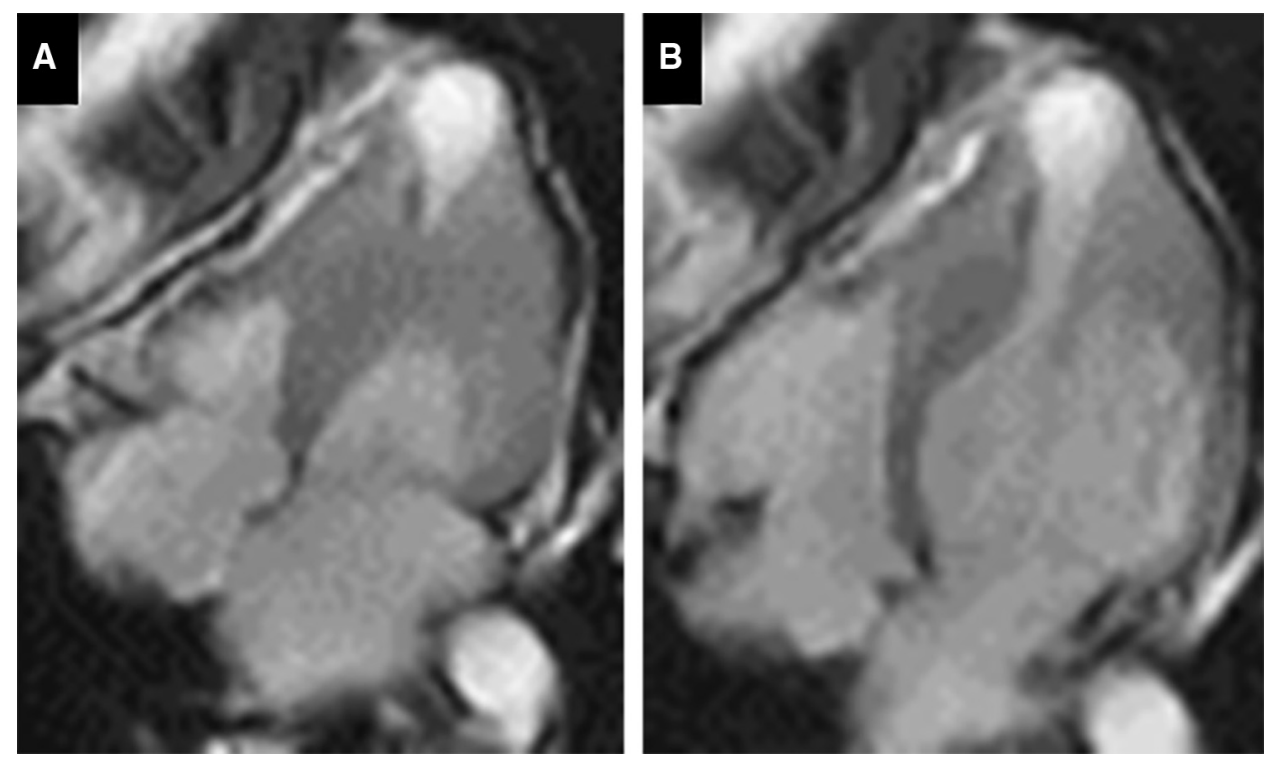

FIGURE 2. Cardiac magnetic resonance imaging showing the presence of an apical aneurysm with midventricular cavity obliteration in (A) systole and (B) diastole.

hypertrophic cardiomyopathy and are associated with worse long-term prognosis. ${ }^{1,2}$ Progression of apical aneurysm was believed to be due to continued subendocardial ischemia from high LV wall stress. This resulted in midventricular obstruction, which then led to higher levels of apical wall stress and ischemia. Resection of the apical aneurysm and midventricular obstruction is a novel surgical treatment for these patients ${ }^{3}$ to improve symptoms by enhancing diastolic filling and to prevent ventricular arrhythmias or thromboembolic complications. This surgical procedure has evolved and can be performed by experienced surgeons with low operative risk and excellent outcomes.

\section{References}

1. Klarich KW, Attenhofer Jost CH, Binder J, Connolly HM, Scott CG, Freeman WK, et al. Risk of death in long-term follow-up of patients with apical hypertrophic cardiomyopathy. Am J Cardiol. 2013;111:1784-91.

2. Maron MS, Finley JJ, Bos JM, Hauser TH, Manning WJ, Haas TS, et al Prevalence, clinical significance, and natural history of left ventricular apica aneurysms in hypertrophic cardiomyopathy. Circulation. 2008;118:1541-9.

3. Kunkala MR, Schaff HV, Nishimura RA, Abel MD, Sorajja P, Dearani JA, et al Transapical approach to myectomy for midventricular obstruction in hypertrophic cardiomyopathy. Ann Thorac Surg. 2013;96:564-70.
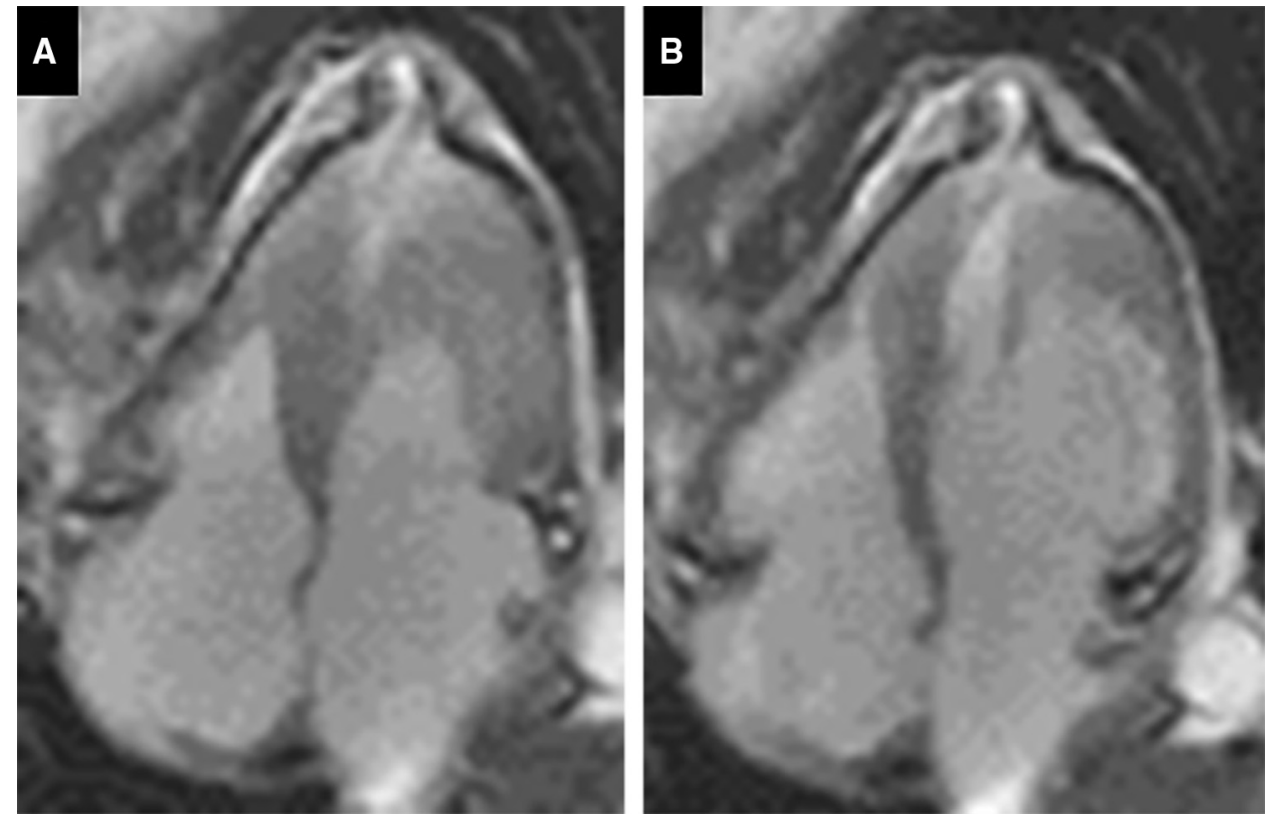

FIGURE 3. Postsurgical cardiac magnetic resonance imaging showing (A) complete resection of the apical aneurysm and (B) enlargement of the left ventricular cavity. 